\title{
The Impact of Virtual Reality Nature Environments on Calmness, Arousal and Energy: a Multi-Method Study
}

\author{
Hildegardo Noronha \\ ITI/LARSyS - University of Madeira \\ Polo Científico e Tecnológico da Madeira, \\ Caminho da Penteada, piso -2 9020-105 Funchal \\ Portugal \\ hildnoronha@gmail.com
}

\author{
Pedro Campos \\ ITI/LARSyS - University of Madeira \\ Polo Científico e Tecnológico da Madeira, \\ Caminho da Penteada, piso -2 9020-105 Funchal \\ Portugal \\ pedro.campos.pt@gmail.com
}

\begin{abstract}
Virtual Reality is the current media's epitome of Immersiveness, Presence and Suspension of Disbelief. Both research and gaming industry communities have been building on this in order to exhaustively research and explore feelings of high-adrenaline, scariness, panic and other visceral and instinctive feelings. We take the opposite approach and try to prove that Virtual Reality can also be used to induce feelings of relaxation and soothingness effectively and strongly. Therefore, it could be used to improve the mental health of people who cannot be exposed to situations that induce said feelings. In our experiments, we found that Virtual Reality can be used to induce a strong sense of Calmness and to reduce the sense of Arousal and Energy, with a high degree of significance, having an effect with short-duration exposures. We also found hints that Virtual Reality may have an effect in the circadian cycle's regulation by exposing the subjects to a virtual sunset.
\end{abstract}

Virtual Reality. Digital Wellbeing. Digital Nature.

\section{INTRODUCTION}

\subsection{Summary}

It is widely agreed that Virtual Reality (VR) is the current media's epitome of Immersiveness, Presence and Suspension of Disbelief. The technology has very appealing characteristics that takes the user experience to a whole new level. To please the vision, it offers a wide field-of-view, stereo-vision, and the ability to look and move your head anywhere inside a virtual environment (with more degrees-of-freedom than is usually possible in other media). It also offers a greater ability to manipulate virtual objects, in three-dimensional spaces, than most media, even though it is still, somewhat, underdeveloped and is, for most commercial solutions, unnatural. Finally, it enables the user to use natural locomotion to navigate the virtual environment which, together with all the previous characteristics, pulls the user into the virtual environment in a way that was never possible before on any other kind of media. All of this creates a sense of Immersiveness, Presence and Suspension of Disbelief so great in some VR experiences and games, making it so visceral, that some people completely forget that they are in a game, which triggers some extreme reactions, including an elevated sense of fear and even panic. These intense feelings have been widely explored by indie developers and is currently making its way into the AAA gaming industry, making it a contributing factor for the widespread adoption of VR. What this paper explores is the polar opposite of the spectrum. Because getting automatic fear and panic reactions out of people is actually something that can be easily achieved in other forms of media (by using, for instance, the typical "jump-scare" cliché), even though it is very intense in VR. For this reason, we explored sensations such as relaxation and that soothing and warm feeling one can get, just like a sunny day at the beach or sitting by a campfire. These feelings have been targeted before by other media, through screen savers in TV sets, computers and mobile phones, but we argue that those feelings achieve a whole new level, viscerallike, when using Virtual Reality - similar to what can be achieved for the panic and fear-like feelings.

By proving that Virtual Reality can effectively create those feelings in a heightened level, the technology can be used in many fields where the mental healthcare needs a boost, from stressful workplaces to healthcare and elderly homes, it could even have beneficial effects in depressions, especially, when people are somewhat limited in the 
locations that can trigger those feelings (such as in big cities, prisons, medical institutions) or when they simply lack the willingness to leave their homes.

\subsection{Research Questions}

Our aim is to discover how effective Virtual Reality is in quickly creating soothing, relaxing, and warm feelings. For this reason, we try to answer the following research questions, taking into account a short time exposure of 1 minute:

(i) Can Virtual Reality strongly relax people?

(ii) Can Virtual Reality strongly increase peoples' mood?

A third research question came up when the data started to be analysed and will be further explained later in the paper:

(iii) Does Virtual Reality have an influence in the circadian cycle?

\section{LITERATURE REVIEW}

Nature is the ultimate therapy. The human, as an integral part of nature, needs it, not only to survive, but also to keep its mental health (Bratman et al., 2019; Parr, 2007). With industrialization and the creation of ever growing cities, sometimes even referred to as concrete jungles, the humans are steadily losing the healing touch of nature (Bratman et al., 2019). This is why the nature tourism is on the rising with its therapeutical values (Buckley, 2020; Buckley and Westaway OAM, 2021), where people can enjoy safaris, hunting trips, cetaceans watching in order to feel closer to nature. Those experiences have, arguably, the same effect as Zoos without having animals removed from their natural habitats, even though they may disturb the animals and cause other issues (Mason, 2000). There are studies pointing that we can get some of the beneficial effects without being, directly in the nature, for instance, while by being indoors. As an example, between many, Philippot (1993), Mcsweeney et al. (2014) and several others (please check Mcsweeney's paper's references for more) explore Indoor Nature Exposure as an alternative to real nature exposure. They review several papers about different key aspects of nature that can be used to improve some aspects of quality of life and both psycho and physical health. From the studies list, we can find good effects coming from potted plants, direct sun-light, a window view. But the effects can arise from more artificial means, such as photographs and videos and even artificial imagery generated by computers.

Older studies indicate good success in inducing a range of feelings using their current media technologies. Philippot (1993) researched the capacity of film segments in, reliably and unequivocally, inducing naturally occurring emotional states on exposed subjects. They exposed the subjects to six short film segments and evaluated their responses by using three questionnaires. They found out that the films can be used to elicit emotions, in a predictable manner, in most subjects. They also found out that the Differential Emotions Scale is better at discriminating between emotional states than the Semantic Differential. Two years later, Gross and Levenson (1995) developed a set of films to elicit eight emotional states (amusement, anger, contentment, disgust, fear, neutral, sadness, and surprise). They selected clips from over 250 films and showed it to 494 English-speaking subjects and then, based on the subjects' responses, selected 2 films for each emotional state.

An evolution into Virtual Reality was just a natural step. In 2003, Plante et al. (2003) studied the possible beneficial psychological effects of doing aerobic exercise while using Virtual Reality. They concluded that Virtual Reality could enhance enjoyment, energy, while reducing tiredness, if used in such a setting. On the other hand, they discovered that Virtual Reality has the opposite effect, if used without the exercise component, by increasing tension and tiredness, and lowering the energy level. One can argue that since this is a 2003 study, the technology has evolved considerably since then and the benefits might have increased while the negative effects might have reduced or been removed altogether. Baños et al. (2005) studied how the immersion affects the sense of presence by comparing Virtual Reality to both a monitor and a projection. They, later, conducted another study (Baños et al., 2006) where they expanded Mood Induction Procedures into Virtual Reality (creating a VR-MIP) and induced different moods (sadness, happiness, anxiety and relaxation) into their experiment subjects by making changes in a Virtual Environment Park. They reported a successful induction in both sadness and happiness using the VR-MIP. Felnhofer et al. (2015) researched the emotional arousalness of Virtual Reality. They studied five emotions (joy, sadness, boredom, anger, and anxiety) by exposing their subjects to an emotionally charged Virtual Park. They found some indications that Presence does not influence emotions in Virtual Reality.

There are also links between Presence and Emotions, in Virtual Reality, as explored by Riva et al. (2007). They explore the ability to elicit emotions in Virtual Reality, like in other medias. They also try to find a relationship between Presence, a strong characteristic in Virtual Reality, and emotions. They confirm the effectiveness of the medium in triggering Anxiety and Relaxing feelings. They found a circular interaction between Presence and Emotions where one inflates the other. 
The same research trend is being expanded into Augmented Reality, as demonstrated by Mehra et al. (2019) whom tried to prove the power of positive mood in the productivity of software developers through the use of Augmented Reality. They tried to improve their working environment by superimposing virtual pets and scenic features unto the real-world - their work environment.

There has been some expansion into the usage of more senses (besides vision and hearing), like demonstrated by Serrano et al. (2016) who ran some experiments using Virtual Reality coupled with touch and smell stimulation in order to induce relaxation. They tested the efficacy of moodinduction procedure in a Virtual Reality (VR-MIP). A high sense of Presence was found and well as a statistical difference in relaxation. They also found no improvement while using smell, but the sense of touch does improve both Presence and relaxation.

Feelings in Virtual Reality have also been researched into a more therapeutically component, as demonstrated by Baus and Bouchard (2014) who reviewed an approach of running exposure therapy, specially phobias, from a Virtual Reality, which they consider to be much more expensive, to Augmented Reality, while still being effective. In Augmented Reality, Juan et al. (2006) developed and tested a prototype using Augmented Reality in order to explore the treatment of acrophobia while exploring the feeling of Presence in immersive photography. They ran parallel tests using a real-world staircase and in immersive photography. A System Usability Scale questionnaire was administered finding out that the sense of Presence was very high in their system but that there was a clear awareness of the Reality versus the Virtual Environment. This context was found to be useful in the treatment of acrophobia. Later, Botella et al. (2010) explored the utilization of Augmented Reality in the treatment of phobias, namely, cockroach phobia. They argue that in vivo exposure is the recommended treatment. They show that Virtual Reality and Augmented Reality is an effective method of treating some of those phobias and show the advantages of using Augmented Reality as a treatment. McLay et al. (2014) studied the effects of Virtual Reality PTSD treatment on Mood and Neurocognitive. They expand the results of PTSD treatment using Virtual Reality into depression and anxiety. They found significant reduction in PTSD and anxiety and significant improvement on emotional Stroop test. There was no improvement in depression nor an improvement in neuropsychologial functions. Herrero et al. (2014) induced Positive Emotions through the usage of Virtual Reality in order to treat Fibromyalgia. Their experiments found no statistical relevant improvement in pain and fatigue related values. But it did show that most of the subjects showed improvements, or no change, in their mood, with only $7.5 \%$ showing some deterioration. They indicate that Virtual Reality is an effective method of treating acute but not chronic pain. In Mental Health, Bermúdez i Badia et al. (2018) proposed an architecture that can foster emotional regulation strategies. The system can generate procedural content based on affection and was rated pleasant by the subjects.

Emotional training can also be achieved by the usage of Virtual Reality, as demonstrated by Bosse et al. (2014) by exploring a system where the military, the law enforcement and other high stress workers can learn to regulate their own emotions. Ferrer-García and Gutiérrez-Maldonado (2011) reviewed the research into how Virtual Reality can be used to treat body image disturbances. They note the lack to published controlled studies in the subject but acknowledge the great potential of Virtual Reality as a substitute for in vivo exposure.

Nature has beneficial effects on humans. Gould van Praag et al. (2017) studied the relaxation and wellbeing effects of Naturalistic environments through autonomic arousal and activation. Their study reinforced the health benefits present in exposure to natural environments. But the effects are beyond health and well-being. Bratman et al. (2012) studied the effect of nature on cognitive function. They reviewed several works and proposed a system to categorize nature experiences. The beneficial effects of nature seem to cross-over to other medias. In this case, to Virtual Reality, as Browning et al. (2020) found out. They researched the effect of 6 minutes $360^{\circ}$ videos of natural settings and found out increased levels of arousal and mood. Yu et al. (2018) expanded beyond nature and also explored urban environments. They found effects on both psychological and physiological values. They found out that the effects of environments in Virtual Reality map those of real life with fatigue levels and several bad feelings increasing in urban settings and decreasing in natural settings. The effects are strong enough to help people recover from stress and anxiety, as demonstrated by Yin et al. (2020) in a bigger 100 subjects study. Instead of pure nature, they went with a biophilic office, but the nature effect is still there.

\section{RESEARCH METHODS}

\subsection{Apparatus}

\subsubsection{Virtual Reality Simulation}

The Virtual Reality Simulation, where the subjects are tested, was built using Unity3D 2019.4, with the High Definition Render Pipeline and the help of the built-in Terrain Tool. The SteamVR Plugin was used to handle the Virtual Reality. Assorted high quality assets from the built-in Asset Store were used to aid on the construction of the environments. 
There are two environments, a beach, and a forest, described below. Each environment has two timeof-the-day, totalling four different combinations that the users can experience.

The experiments were built with as much visual quality as it was possible, given our time, human and financial constraints.

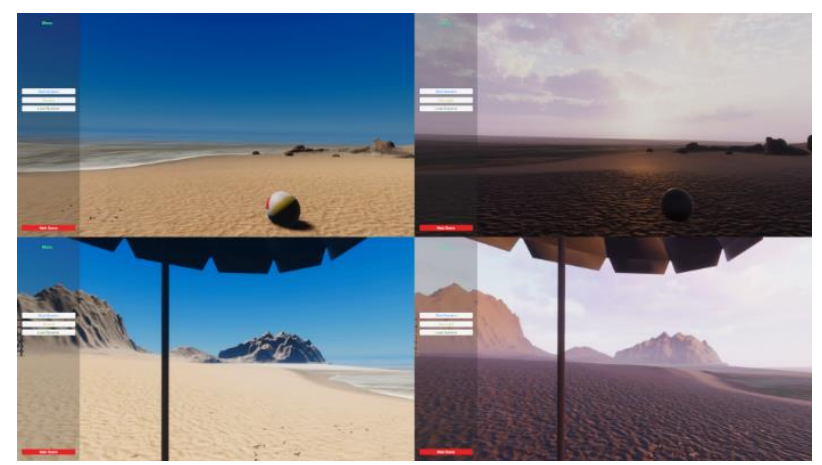

Figure 1: The Beach scenery. Midday on the left; sunset at the right

(i) Beach - A golden-sand beach, near the ocean. Some palm trees paint the sand dunes behind the subjects. Assorted beach-related things are spread around the subject. The sound of waves can be heard coming from the sea. These sounds do not change between different times of the day. There are two time-of-the-day settings that the subjects can experience:

a. At midday - a very high strong sun.

b. At sunset - a romantic sunset.

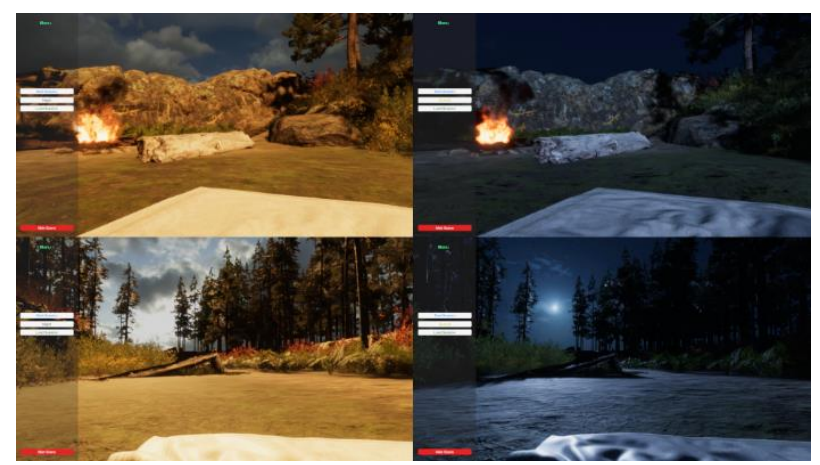

Figure 2: The Forest scenery. Afternoon on the left; night at the right.

(ii) Forest - A calm, tree-filled, forest. The subjects are in a small sunken glade with are built nearby. You can hear the re going and a slight breeze on the trees. These sounds do not change between different times of the day. Just like in the beach, there are two time-of-the-day settings that the subjects can experience:

a. During the afternoon - the sun is comfortably midway in the afternoon creating some longish shadows while keeping good visibility.

b. During the night - the sun is long gone, giving space to a starry sky and the moon.

\subsubsection{Virtual Reality Hardware}

We used the HTC Vive Pro as the Virtual Reality headset. No controllers are used since there is no direct user interaction, other than looking around.

\subsubsection{Sensors}

We used a MUSE $S$ to sense the level of relaxation of the user. It is a headband that uses Electroencephalography to monitor brain activity and outputs blackboxed data, such as level of relaxation. We also used a generic Heart-Rate sensor band to monitor the subjects' heart rate.

\subsubsection{Computer}

Due to the high requirements of the current generation Virtual Reality, we used a high-end gaming computer composed of an Intel i7-9700K CPU and a Nvidia GeForce RTX2080 Graphics Card. The rest of the computer was built with relevant matching high-end components.

\subsection{Questionnaires}

To complement the data gathered by the sensors, we exposed the subjects to the following standard questionnaires: 1) the AD ACL (Thayer, 1986) and 2) the SAM(Bradley and Lang, 1994). From all the questionnaires that we explored, we found these two to better evaluate the more positive and calm feelings that we are trying to study. The other related questionnaires are more focused on active and negative feelings.

We also exposed the subjects to another two nonstandard, Likert-scale questionnaires - one at the beginning of the experiment and another at its conclusion.

\subsubsection{Activation-Deactivation Adjective Check List (AD ACL)}

The AD ACL that we used is based on Thayer's original questionnaire (Thayer, 1986). It has the original 20 adjectives randomly distributed in order not to influence the subjects' answers and to reduce coupling. The remaining of the questionnaire is standard with the standard 4 levels that the subject can feel.

\subsubsection{Self-Assessment Manikin (SAM)}

We used a standard SAM (Bradley and Lang, 1994) questionnaire with drawn manikins and the standard 9 levels scale but limited to Valence and Arousal.

\subsubsection{Pre-Questionnaire}

The pre-questionnaire tries to assess the prior level of experience of the subjects in Technology, 
Gaming and Virtual-Reality. It uses standard 7 points Likert-scale. All the 3 questions range from 1 - Low to 7 - High with a middle point at 4 - Medium.

\subsubsection{Post-Questionnaire}

The post-questionnaire tries to assess how the subject feels about the subject in a subjective manner. It also uses standard 7 points Likertscales. All the 4 questions range from 1 - Not at all to 7 - $A$ Lot. The questionnaire asks the subject the following questions:

(iii) I feel that the Virtual Reality environments relaxed me.

(iv) I would enjoy spending more time relaxing in Virtual Reality environments.

(v) I feel that, relaxing in a Virtual Reality environment could substitute a real-world alternative, in situations where the realworld alternatives are not accessible (e.g.: prisons, remote locations, big concrete cities).

(vi) I would pay to enjoy spending time relaxing in a Virtual Reality environment.

The questionnaire also asks them to sort the experiments by order of relaxation.

\subsection{EXPERIMENTAL PROTOCOL}

\subsubsection{The Experiment Script}

\section{Pre-Experiment:}

The experiment starts with the subjects being asked to fill-in the pre-questionnaire followed by the evaluation of the subjects' current mood. The mood is evaluated using the $A D A C L$ and the $S A M$ questionnaires. This establishes a baseline, where the subject is not yet exposed to the Virtual Reality. The two sensors (Muse and Heart-rate sensor band) are then set up on the subject. The subject is, then, asked to sit quietly on a chair, facing the wall in a silent room, for 2 minutes (1 for the calibration of the MUSE $S$ and another for the data gathering). We use this to set up another baseline, this time, for the sensors (mental relaxation and rest heart rate), again, before exposure.

\section{Main Experiment:}

The subject is exposed to each of the 4 Virtual Reality experiences random order. It starts with 1 minute of calibration of the MUSE $S$ followed by 1 minute of exposure to the experience. After each experience, the subject is asked to, again, fill up the $A D A C L$ and the SAM questionnaires, allowing us to assess their mood after each iteration. These 4 iterations are also experienced in the same chair, for the same 1 minute and without moving.

\section{Post-Experiment:}

In the end, the subject repeats the baseline measurements to allow us to compare the before and after of the experiment. The subject is finally asked to fill up the post-questionnaire before concluding the experiment.

\section{Duration:}

The experiment takes about 30 minutes per user. There are two short pre-questionnaires that are then followed by a baseline "empty" experience that takes a little more than 2 minutes ( 1 min calibration +1 min experience). The main experiment takes $(1 \mathrm{~min}$ calibration +1 min experience) $\times 4$ experiments $=8$ minutes. All of this is followed by another baseline "empty" experience and the post-questionnaires. This all adds up to about 12 minutes of controlled time plus the questionnaires and overheads.

\section{Clarifications:}

Note that the subject is asked to remain still, when being evaluated, to avoid the subject's movements to affect the heart rate. This way, any change on the subject's heart rate can be attributed to a mental state, instead of a physical movement.

Also note that the order of the 4 iterations are picked from a previously built list. The list contains all the possible 24 permutations. The users are then, attributed, sequentially until all the permutations are tested. The list is, then, restarted.

Finally, the 1-minute calibration was chosen because the MUSE S was taking somewhat below 1 minute to calibrate. In order to keep the user's experiences as similar to each other as possible, we decided to calibrate for 1 minute, even if the MUSE S was done calibrating before that. As for the 1-minute Virtual Reality experiences, please check Section Limitations and Future Work.

\subsection{Subjects}

A total of 29 subjects participated in the experiments, 20 males and 9 females. Ages ranged from 21 to 39.7 subjects had no to little experience with Virtual Reality, 18 had moderate experience with Virtual Reality and 4 had a lot of experience with Virtual Reality. The subjects were asked out from anyone in-campus (students, professors, staff, researchers, other), including the local university, research institutes and supporting buildings. A small number of out-of-campus subjects were also used.

\section{RESULTS}

We conducted paired samples t-tests comparing the baseline, measured before the subjects are exposed to the experiment, to each of the four environment type (Beach at Noon, Beach at Sunset, Forest at Afternoon, Forest at Night). The order of the iterations is randomized to avoid any order effects. We also compared the baseline to 
each iteration (1st, 2nd, 3rd, and 4th), regardless of what environment it was.

We compared the baseline to the average of the 4 iterations, to the average of each environment (considering both times of the day) and, finally, against the after-exposure baseline (where relevant). We also calculated some simple averages, standard deviations, the effect power, and the effect size, where relevant. All this data is on several tables of the relevant subsections. Figure 3 shows a high-level view of the results, before and after the exposure. Figure 5 in Annex also shows a box-and-whiskers chart of the data to better clarify the data's distribution.

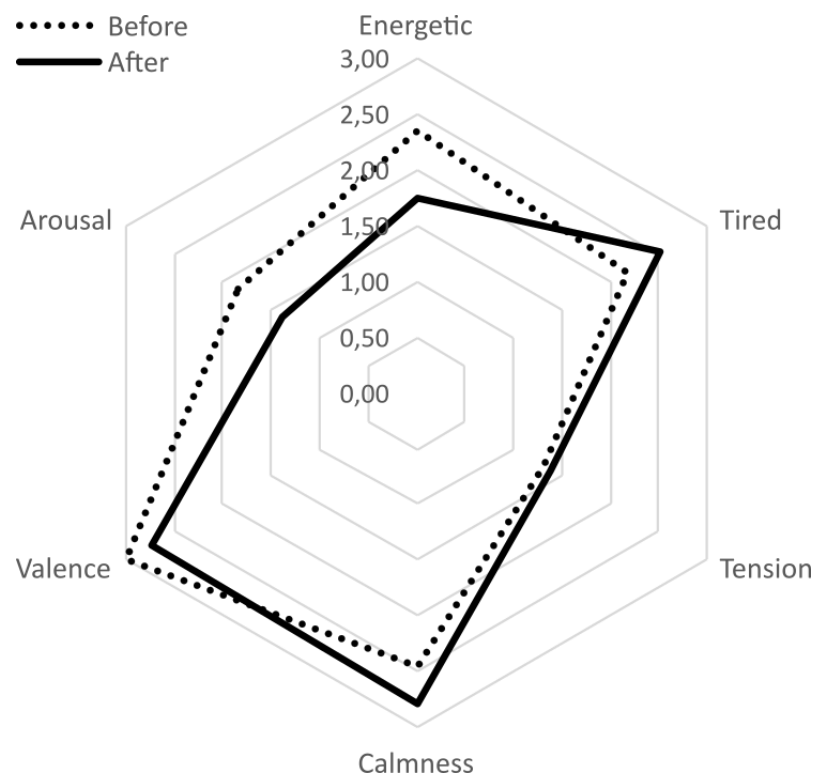

Figure 3: The feelings of the subjects, Before and After exposure

\subsection{Activation-Deactivation Adjective Check List (AD ACL) Questionnaire}

The AD ACL Questionnaire merges the adjectives into 4 feelings: Energetic, Tired, Tension and Calmness, that we use to further our research. Our Research Questions point to an increase in Calmness. We make no assumptions as to Energy, Tired and Tension other than there may be an effect. As such, we have 4 hypotheses: 1) The Calmness value of exposed subjects should increase; 2) The Energy value of exposed subjects should be different; 3 ) The Tired value of exposed subjects should be different; 4) The Tension value of exposed subjects should be different.

Hypothesis 1 (Calmness) is statistically significant for the Beach Midday and Sunset but not for the Forest. It has p-values of 0.009513 and 0.000024 , respectively. The increase in Calmness is of 0.36 and 0.52 , respectively, and in a scale of 1 to 4 . It also an intermediate to large effect size with values of 0.529 to 0.929 .
Note that the next 4 tables have the following headers: Average rating; Standard Deviation; Difference vs. Base before exposure; $p$-value of the t-test; Power; Effect Size.

Table 1: Calmness.

\begin{tabular}{|l|c|c|c|c|c|c|}
\hline Measure. & $\boldsymbol{\mu}$ & $\boldsymbol{\sigma X}$ & $\boldsymbol{\Delta}$ & $\mathbf{p}$ & Power & E.S. \\
\hline $\begin{array}{l}\text { Base- } \\
\text { Before }\end{array}$ & 2.5 & 0.5 & - & - & - & - \\
\hline $\begin{array}{l}\text { Beach; } \\
\text { Midday }\end{array}$ & 2.9 & 0.6 & 0.4 & 0.01 & 0.99049 & 0.53 \\
\hline $\begin{array}{l}\text { Beach; } \\
\text { Sunset }\end{array}$ & 3.1 & 0.5 & 0.5 & $\begin{array}{l}0.00 \\
002\end{array}$ & 0.99998 & 0.93 \\
\hline $\begin{array}{l}\text { Forest; } \\
\text { Afternoon }\end{array}$ & 2.6 & 0.6 & 0.1 & 0.24 & - & - \\
\hline $\begin{array}{l}\text { Forest; } \\
\text { Night }\end{array}$ & 2.5 & 0.7 & -0.0 & 0.40 & - & - \\
\hline
\end{tabular}

Hypothesis 2 (Energy) is statistically significant for all but the Forest at Night. It has p-values of $0.002103,0.000043,0.005301$. The Energetic values always decreases ranging from 0.34 to 0.67 , in a scale of 1 to 4 . The effect sizes, however, are adverse for all.

Table 2: Energetic.

\begin{tabular}{|l|c|c|c|c|c|c|}
\hline Measure. & $\boldsymbol{\mu}$ & $\boldsymbol{\sigma X}$ & $\boldsymbol{\Delta}$ & $\boldsymbol{p}$ & Power & E.S. \\
\hline $\begin{array}{l}\text { Base- } \\
\text { Before }\end{array}$ & 2.5 & 0.7 & - & - & - & - \\
\hline $\begin{array}{l}\text { Beach; } \\
\text { Midday }\end{array}$ & 2.2 & 0.8 & -0.3 & $\begin{array}{l}0.00 \\
210\end{array}$ & 0.99789 & -0.69 \\
\hline $\begin{array}{l}\text { Beach; } \\
\text { Sunset }\end{array}$ & 1.7 & 0.6 & -0.7 & $\begin{array}{l}0.00 \\
004\end{array}$ & 0.99996 & -0.88 \\
\hline $\begin{array}{l}\text { Forest; } \\
\text { Afternoon }\end{array}$ & 2.0 & 0.8 & -0.5 & $\begin{array}{l}0.00 \\
530\end{array}$ & 0.99470 & -0.60 \\
\hline $\begin{array}{l}\text { Forest; } \\
\text { Night }\end{array}$ & 2.3 & 0.8 & -0.2 & 0.24 & - & - \\
187 & & \\
\hline
\end{tabular}

Hypothesis 3 (Tired) is statistically significant for the Beach at the Sunset. It has a p-value of 0.00078 . The Tired value increases 0.5143 , in a scale of 1 to 4. Its effect size is medium with a value of 0.7 .

Table 3: Tired.

\begin{tabular}{|l|c|c|c|l|l|l|}
\hline Measure. & $\boldsymbol{\mu}$ & $\boldsymbol{\sigma X}$ & $\boldsymbol{\Delta}$ & $\mathbf{p}$ & Power & E.S. \\
\hline $\begin{array}{l}\text { Base- } \\
\text { Before }\end{array}$ & 1.9 & 0.8 & - & - & - & - \\
\hline $\begin{array}{l}\text { Beach; } \\
\text { Midday }\end{array}$ & 2.1 & 0.8 & 0.2 & $\begin{array}{l}0.10 \\
784\end{array}$ & - & - \\
\hline $\begin{array}{l}\text { Beach; } \\
\text { Sunset }\end{array}$ & 2.5 & 0.7 & 0.5 & $\begin{array}{l}0.00 \\
078\end{array}$ & 0.99922 & 0.7 \\
\hline $\begin{array}{l}\text { Forest; } \\
\text { Afternoon }\end{array}$ & 2.2 & 0.8 & 0.3 & 0.10 & - & - \\
\hline $\begin{array}{l}\text { Forest; } \\
\text { Night }\end{array}$ & 2.0 & 0.8 & 0.0 & $\begin{array}{l}0.93 \\
538\end{array}$ & - & - \\
\hline
\end{tabular}

Hypothesis 4 (Tension) is statistically significant for the Forest at Night. It has a p-value of 0.044 and an increase in Tension value of 0.31 , in a scale of 1 to 4. It has a medium effect size of 0.747 . 
Table 4: Tension

\begin{tabular}{|l|c|c|c|c|l|l|}
\hline Measure. & $\boldsymbol{\mu}$ & $\boldsymbol{\sigma X}$ & $\boldsymbol{\Delta}$ & $\mathbf{p}$ & Power & E.S. \\
\hline $\begin{array}{l}\text { Base- } \\
\text { Before }\end{array}$ & 1.3 & 0.3 & - & - & - & - \\
\hline $\begin{array}{l}\text { Beach; } \\
\text { Midday }\end{array}$ & 1.3 & 0.3 & -0.1 & 0.527 & - & - \\
\hline $\begin{array}{l}\text { Beach; } \\
\text { Sunset }\end{array}$ & 1.2 & 0.3 & -0.1 & 0.277 & - & - \\
\hline $\begin{array}{l}\text { Forest; } \\
\text { Afternoon }\end{array}$ & 1.4 & 0.5 & 0.0 & 0.734 & - & - \\
\hline $\begin{array}{l}\text { Forest; } \\
\text { Night }\end{array}$ & 1.6 & 0.8 & 0.3 & 0.044 & 0.956 & 0.75 \\
\hline
\end{tabular}

\subsection{Self-Assessment Manikin (SAM) Questionnaire}

The SAM questionnaire evaluates Valence and Arousal. We consider the scale to go from -4 to +4 , with 0 being a neutral value. Our Research Questions point to a decrease in Arousal. We make no assumptions as to Valence. As such, we have 2 hypotheses: 1) The Arousal value of exposed subjects should be lower; 2) The Valence value of exposed subjects should be different.

Hypothesis 1 (Arousal) is statistically significant for the Beach (both times-of-day), the Forest at the afternoon and the Average of all experiments. It has $p$-values of $0.00073,0.00010,0.04547$ and 0.00240 . The decrease in Arousal values ranges from 0.97 to 1.46 . All the effect sizes are adverse.

Table 5: Arousal

\begin{tabular}{|l|c|c|c|c|c|c|}
\hline Measure. & $\boldsymbol{\mu}$ & $\boldsymbol{\sigma X}$ & $\boldsymbol{\Delta}$ & $\boldsymbol{p}$ & Power & E.S. \\
\hline $\begin{array}{l}\text { Base- } \\
\text { Before }\end{array}$ & -0.3 & 2.6 & 2.6 & - & - & - \\
\hline $\begin{array}{l}\text { Beach; } \\
\text { Midday }\end{array}$ & -1.5 & 2.1 & -1.2 & 0.00 & 0.99927 & -0.34 \\
\hline $\begin{array}{l}\text { Beach; } \\
\text { Sunset }\end{array}$ & -1.7 & 2.1 & -1.5 & $\begin{array}{l}0.00 \\
010\end{array}$ & 0.99990 & -0.38 \\
\hline $\begin{array}{l}\text { Forest; } \\
\text { ffternoon }\end{array}$ & -0.9 & 2.2 & -0.6 & $\begin{array}{l}0.04 \\
547\end{array}$ & 0.95453 & -0.17 \\
\hline $\begin{array}{l}\text { Forest; } \\
\text { Night }\end{array}$ & -0.5 & 2.3 & 0.2 & $\begin{array}{l}0.27 \\
068\end{array}$ & - & - \\
\hline
\end{tabular}

Hypothesis 2 (Valence) has no statistically significance in any of the experiences.

Table 6: Valence

\begin{tabular}{|l|c|c|c|c|l|l|}
\hline Measure. & $\boldsymbol{\mu}$ & $\boldsymbol{\sigma X}$ & $\boldsymbol{\Delta}$ & $\mathbf{p}$ & Power & E.S. \\
\hline $\begin{array}{l}\text { Base- } \\
\text { Before }\end{array}$ & 2.0 & 1.5 & - & - & - & - \\
\hline $\begin{array}{l}\text { Beach; } \\
\text { Midday }\end{array}$ & 2.1 & 1.6 & 0.1 & 0.69 & - & - \\
\hline $\begin{array}{l}\text { Beach; } \\
\text { Sunset }\end{array}$ & 2.2 & 1.4 & 0.2 & 0.48 & - & - \\
\hline $\begin{array}{l}\text { Forest; } \\
\text { Afternoon }\end{array}$ & 1.9 & 1.5 & -0.1 & 0.54 & - & - \\
\hline $\begin{array}{l}\text { Forest; } \\
\text { Night }\end{array}$ & 1.6 & 1.5 & -0.4 & 0.20 & - & - \\
\hline
\end{tabular}

\subsection{Brain Relaxation}

The hardware that we used - the MUSE S - has an app that outputs 3 values, measured along time: Active, Neutral, Relaxed. To merge the 3 values into 1 , and since we are aiming at relaxation, we subtracted the Active time from the Relaxed time to find out the adjusted relaxed time (in 1 minute).

Our hypothesis is "The relaxed time should be higher after exposure". We achieved statistically significant results when comparing the baseline after exposure with the baseline before exposure as well as with the same comparison with the Beach. The p-values of the t-test are 0.0122 and 0.0131 , respectively, with an increase of 8.79 and 9.32 seconds of relaxation in a minute of exposure. The effect sizes are small with values of 0.414 and 0.37 , respectively.

Table 7: Brain Relaxation (in seconds)

\begin{tabular}{|l|l|l|l|l|l|l|}
\hline Measu. & $\boldsymbol{\mu}$ & $\boldsymbol{\sigma X}$ & $\boldsymbol{\Delta}$ & $\mathbf{p}$ & Power & E.S. \\
\hline $\begin{array}{l}\text { Base- } \\
\text { Before }\end{array}$ & 21 & 18 & - & - & - & - \\
\hline Beach & 31 & 11 & 9.3 & 0.0131 & 0.9869 & 0.37 \\
\hline Forest & 27 & 14 & 5.9 & 0.1119 & - & - \\
\hline $\begin{array}{l}\text { Beach }+ \\
\text { Forest }\end{array}$ & 28 & 9 & 6.9 & 0.0437 & 0.9563 & 0.263 \\
\hline $\begin{array}{l}\text { Base- } \\
\text { After }\end{array}$ & 30 & 15 & 8.8 & 0.0122 & 0.9878 & 0.414 \\
\hline
\end{tabular}

\subsection{Heart rate}

We take the median of the heart rate of each of the 6 measurements (before exposure, 4 virtual reality environments, after exposure). We use the median, instead the average, to filter out small spikes in the heart rate that occur due to subject movements. We then use these values to conduct the t-tests.

Our hypothesis is "The Heart-Rate of the subjects should be lower after the exposure". We were unable to prove or disprove this hypnotises with our data. The data fluctuates around no change with no statistically significant values.

\section{Table 8: Heart Rate}

\begin{tabular}{|l|c|l|l|l|l|l|}
\hline Measure. & $\boldsymbol{\mu}$ & $\boldsymbol{\sigma X}$ & $\boldsymbol{\Delta}$ & $\mathbf{P}$ & Power & E.S. \\
\hline $\begin{array}{l}\text { Base- } \\
\text { Before }\end{array}$ & 73 & 10 & - & - & - & - \\
\hline $\begin{array}{l}\text { Beach; } \\
\text { Midday }\end{array}$ & 73 & 9 & 0.4 & 0.35 & - & - \\
\hline $\begin{array}{l}\text { Beach; } \\
\text { Sunset }\end{array}$ & 72 & 9 & -0.5 & 0.29 & - & - \\
\hline $\begin{array}{l}\text { Forest; } \\
\text { Afternoon }\end{array}$ & 73 & 9 & 0.3 & 0.36 & - & - \\
\hline $\begin{array}{l}\text { Forest; } \\
\text { Night }\end{array}$ & 74 & 9 & 0.6 & 0.25 & - & - \\
\hline $\begin{array}{l}\text { Base- } \\
\text { After }\end{array}$ & 73 & 8 & 0.1 & 0.47 & - & - \\
\hline
\end{tabular}

\subsection{Pre-Questionnaire}

We did a simple average of all the subjects answers to the pre-questionnaire. The average 
value for Technology Experience if of 5.68, for Gaming Experience is of 4.36 and for Virtual Reality is of 3.46. The range of it goes from 1 - Low to 7 - High with a middle point at 4 - Medium.

\subsection{Post-Questionnaire}

We did a simple average of all the subjects' answers to the post questionnaire. The subjects answered an average of 5.18 as to feeling that the Virtual Reality environments relaxed them, 5.07 as to whether they would enjoy spending more time relaxing in the Virtual Environments, 5.32 that Virtual Reality could be used as a substitute for the real-world in situations where the subject has no access to relaxing alternatives, and 3.32 as to they would pay to enjoy relaxing in a Virtual Environment. The range of it goes from 1 - Not at all to 7 - A Lot. The questions (Q1 through Q4) can be found in A.2.

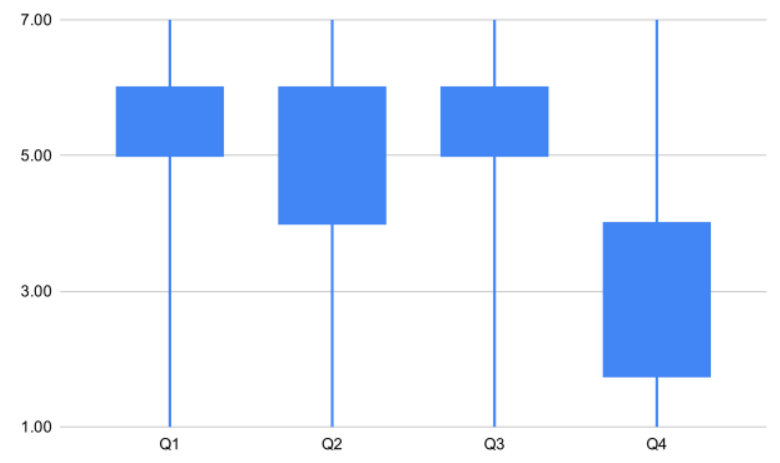

Figure 4: Answers to the Post-Questionnaire.

\section{Discussion}

Our tests confirmed, with a very high degree of statistically significance (best $p=0.000024$; Beach at Sunset), that Nature Scenes in Virtual Reality can induce a sense of Calmness in the subjects who were exposed to it. The effect is considerably strong (with a power of 0.999976 and an effect size of 0.929): the subjects report an average increase in Calmness of 0.5214 points (Beach at Sunset) in a scale ranging from 1 to 4 (AD ACL). This effect is felt even in sessions of very short duration (1 minute). There is also a decrease in the sense of Energy, consistent with the current (Plante et al., 2003), with a high degree of statistically significance $(p=$ 0.000043; Beach Sunset). This effect is even stronger than the Calmness, with a decrease of 0.67 points (Beach at sunset) but its effect size is, actually, adverse (negative). The Tired feeling is only statistically significant for the Beach at sunset $(p=$ 0.00078 ) with an increase of 0.5143 points. It has an effect size of 0.7 . The Tension feeling has no statistically significant except for the Forest at Night $(p=0.044)$. Its effect size is 0.747 . The subjects felt uneasy - scared even - and this can be seen in an increase of 0.3071 points in Tension. Some subjects even commented it directly that they were scared and/or were waiting for some kind of jump-scare. It was also rated, by most subjects, as the least relaxing of the 4 experiments. Note that the nature of the experiment was not explained to them, so they did not know that we were looking into relaxation instead of fear, and, as said before, some subjects were even expecting some kind of jump-scare.

The data supports that there is a statistically significant (best $p=0.00010$, Beach at Sunset) decrease of 1.46 points in Arousal, in a scale ranging from -4 to +4 , in the Beach experiment. The other scenarios also have a statistically significant reduction in Arousal except for the Forest at Night. All the effect sizes are adverse. The Valence shows no statistically significant data. Also, there seems to be little to no change in Valence, despite the lack of statistically significance. However, the Forest at night shows a decrease of 0.39 , but not statistically significant $(p=0.20)$.

The MUSE $S$ shows statistically significant results for both the Beach and the second baseline, after exposure, exhibiting the respective $p$-values of 0.013 and 0.012 . It shows a considerable increase in relaxation time of 9.32 and 8.79 seconds (in 1 minute; from a baseline of 21.21 seconds before exposure). It has small effect sizes. This seems to indicate that the effect is maintained even after the users are no longer exposed. We did not, however, measured for how long this effect lasts.

The Heart-rate data did not produce any statistically significant data. There is a slight oscillation, below 1 BPM, and the p-value denies us any useful conclusions.

The subjective tests point to a higher than average feeling of Relaxation with 5.18 points in a 7-point Likert scale. Subjects would also like to relax more, in Virtual Reality (5.07 points). This does seem to point to the fact that the subjects feel a good level of Relaxation during the experiments - enough for them to feel the difference, in a conscious level. Some subjects also expressed this feeling with their words pointing to both a high level of relaxation and even sleepiness, despite being alone on a room with strangers performing an experiment. But we are aware that, this being a subjective questionnaire and a matter of opinion, our low number of answers might not be representative of the population. This leads us to the next question where they give their opinion on whether or not they feel like a system like this could substitute the reality in situations where it is not possible to experience similar environments (like prisons, remote locations, big concrete cities). This may be interpreted as a loaded question and, as such, appears after the other questions to avoid directing the subjects. The subjects did answer a little bit higher - 5.32 - but still similar to the other two questions. We built the three questions to evaluate almost the same thing with, somewhat, different 
phrasing. And the subjects did stay around the mid 5 s.

The last question asks if the subjects were willing to pay to experience more relaxation in Virtual Reality environments. Now the value does go down, as expected, to 3.32 , in the same scale. This may be interpreted as an opinion on how important they feel a system like this is, despite its capabilities to perform. We feel that 3.32 is still high enough, in light of the other answers and all the experiment results. Hence, their true feeling about relaxing in Virtual Reality, might be between the $3 \mathrm{~s}$ and the $5 \mathrm{~s}$, pointing at a mediumstrength subjective feeling.

\section{Conclusion}

Our experiments indicate a statistically significant and strong increase in Calmness and a decrease in Energy. There is also a statistically significant and strong decrease in Arousal. This is also confirmed by the subject data where the subjects feel above average relaxation (greater than 5 in a 7-point Likert scale with a central neutral point at 4) coupled with some verbal, free-form indications of such. All this data confirms our hypothesis that "Virtual Reality can strongly relax people".

The same data does not provide any statistically significant information about Valence so we will have to withdraw any conclusions about the hypothesis that "Virtual Reality strongly increase peoples' mood".

The hypothesis that "Virtual Reality help to regulate the circadian cycle" is, partially, confirmed. At sunset (a synchronization time for the circadian cycle), the data showed a statistically significant increase in Tired and a decrease in Energy. It also showed a statistically significant increase in Calmness and a statistically significant reduction in Arousal. These changes are also stronger in the sunset that they were for the remain of the experiments, and the 4 were felt all at the same time during the sunset. We do not make a stronger position in confirming this because we only explored a small portion of the circadian cycle. But the data does show an influence at the (virtual) sunset.

An unforeseen and unfortunate conclusion is that our subjects did not enjoy being in a Forest at night, despite it being under the full moon with a fire going nearby. There is a big decrease in Valence, even if not statistically significant, and a statistically significant $(p=0.044)$ moderate increase in Tension. The subjects corroborated this verbally. Unfortunately, this may have reduced the relaxation capabilities of the experiment as a whole, especially for the subjects who felt fear. (Boyce et al., 2000) points to about 30 lux being enough for the perception of safety, at least in city settings. In exceptional conditions, the moon can reach up to 32 lux. But typical values are way below those values, which is below the safety threshold. Regardless, we cannot guarantee the output of the head mounted display. At this point, we can only speculate that the lux values may not be the only factor and the forest setting and/or the absence of other humans may be what is actually causing these feelings. It can even have a cultural explanation, as (Dunn and Edensor, 2020) explores.

All of this confirms that Virtual Reality with nature scenarios, can be used to effectively induce a strong feeling of relaxation in people. There are also indications of a possible effect in the circadian cycle.

\section{LIMITATIONS AND FUTURE WORK}

\subsection{Sound}

We did not consider the effect of the sound. The sound, by itself can cause effects that could interfere and conflict with our results. This should be addressed in the future by either comparing the effects of sound vs no sound or even completely remove the sound from the experiment. Different sounds could also be explored.

\subsection{Comparing with the real life}

We did not compare the Virtual Reality against the real life. The idea was considered but was discarded for being too difficult and expensive to take users to a beach and a forest. It would also be nearly impossible to control all the variables due to external influences (including people external to the experience).

\subsection{Extra props}

Having the users sit on a picnic towel or in a beach stretcher, just like it is represented on the Virtual Reality environment, could have created a stronger effect. This could be interesting to explore since the haptic feedback could add to the effects of the experiences.

\subsection{Users Background}

We only realized how important the users' background could affect the experiment when a user noted that he really liked the beach scenario due to his childhood. Unfortunately, by this time, it was already too late to fix the experiment protocol.

\subsection{More Sceneries}

We studied the effects of just two natural environments. More types of natural and even artificial environments could give us different results. One could expect that some environments would be more appealing to different people with different backgrounds and experiences. 


\subsection{The Heart rate}

We limited the users to sitting still, during the experiences. This was a limitation that we set up due to the changes that simply moving around can do to the heart rate. Unfortunately, this can also interfere with our results. On one side, the users are artificially "locked-in-place" and may feel less relaxed and can even break immersion. On the other side, moving around freely could create more variable experiences for each user which would be harder to control and measure.

\subsection{One-minute experiences}

The choice of 1 minute of Virtual Reality experience is an arbitrary one and we did not base it in any literature. We did, however, choose the time to make up the experience time long enough to expose the users as much as possible without taking too long and negatively affect their feelings (boredom, sleepiness, willing to quit the experiment). We ended up with the value of 1 minute by running some pre-tests and trying to find out when the user was started to get bored with the experiences. With our short, informal sample, most users felt that 2 minutes was a bit too long but were perfectly fine with a 1-minute Virtual Reality experiment.

\subsection{MUSE S}

The MUSE S equipment used on this study had some technical issues that hindered the experiment. Namely, the calibration was mandatory every time we would log data and it would take, up to 1 minute. We encountered some users (usually with thicker and/or longer hair) that would make the MUSE S very hard to calibrate, prolonging the session behind what should be.

\subsection{Sample size and protocol refinement}

We feel that the whole study could benefit from a bigger sample size. Even though we did find some interesting results, a bigger sample size could definitely make a stronger case. The experiment protocol could also benefit from a refinement with the experience we gathered on this study.

\subsection{Circadian cycle and the night}

We, unintentionally, found effects that are, possibly, related to the circadian cycle at the sunset and even at night. This could indicate that Virtual Reality is strong enough to even affect it and it is an interesting venue to explore in the future.

\section{A. ANNEX}

\section{A.1. Data Distribution}

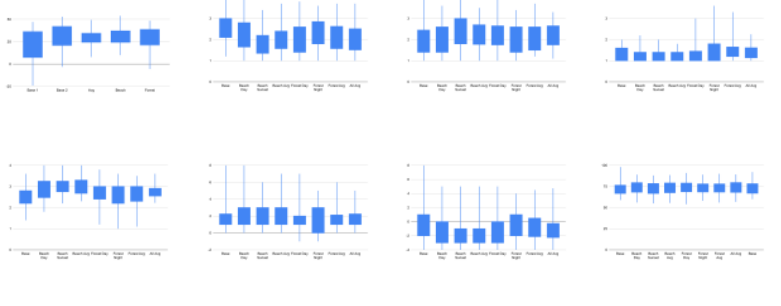

Figure 5: Data's Distribution

From Left to Right, Top to Bottom:

a - Muse: Pre-Baseline; Post Baseline; Experiments Average; Beach; Forest.

b - Energetic: Baseline; Beach Day; Beach Sunset; Beach Average; Forest Day; Forest Night; Forest Average; Experiments Average.

c - Tired: Baseline; Beach Day; Beach Sunset; Beach Average; Forest Day; Forest Night; Forest Average; Experiments Average.

d - Tension: Baseline; Beach Day; Beach Sunset; Beach Average; Forest Day; Forest Night; Forest Average; Experiments Average.

e - Calmness: Pre-Baseline; Post Baseline; Experiments Average; Beach; Forest.

f - Valence: Baseline; Beach Day; Beach Sunset; Beach Average; Forest Day; Forest Night; Forest Average; Experiments Average.

g - Arousal: Baseline; Beach Day; Beach Sunset; Beach Average; Forest Day; Forest Night; Forest Average; Experiments Average.

h - Heart rate: Pre-Baseline; Beach Day; Beach Sunset; Beach Average; Forest Day; Forest Night; Forest Average; Experiments Average; Post Baseline.

\section{A.2. Post-Questionnaire}

Q1.I feel that the Virtual Reality environments relaxed me.

Q2.I would enjoy spending more time relaxing in Virtual Reality environments.

Q3.I feel that, relaxing in a Virtual Reality environment could substitute areal-world alternative, in situations where the real-world alternatives are not accessible (e.g.: prisons, remote locations, big concrete cities).

Q4.I would pay to enjoy spending time relaxing in a Virtual Reality environment.

\section{ACKNOWLEDGMENTS}

This research was partially funded by IDERAM through grants no. M1420-01-0247-FEDER-000019 and M1420-01-0247-FEDER-00003 


\section{REFERENCES}

Baños, R., Botella, C., Alcañiz Raya, M., Liaño, V., Guerrero, B., Rey, B., 2005. Immersion and Emotion: Their Impact on the Sense of Presence. Cyberpsychology \& behavior : the impact of the Internet, multimedia and virtual reality on behavior and society 7 , 734-41. https://doi.org/10.1089/cpb.2004.7.734

Baños, R.M., Liaño, V., Botella, C., Alcañiz, M., Guerrero, B., Rey, B., 2006. Changing Induced Moods Via Virtual Reality, in: IJsselsteijn, W.A., de Kort, Y.A.W., Midden, C., Eggen, B., van den Hoven, E. (Eds.), Persuasive Technology, Lecture Notes in Computer Science. Springer, Berlin, Heidelberg, pp. 7-15. https://doi.org/10.1007/11755494_3

Baus, O., Bouchard, S., 2014. Moving from Virtual Reality Exposure-Based Therapy to Augmented Reality Exposure-Based Therapy: A Review. Front. Hum. Neurosci. 8. https://doi.org/10.3389/fnhum.2014.00112

Bermúdez i Badia, S., Quintero, L., Cameirão, M., Chirico, A., Triberti, S., Cipresso, P., Gaggioli, A., 2018. Toward Emotionally Adaptive Virtual Reality for Mental Health Applications. IEEE Journal of Biomedical and Health Informatics PP, 1-1. https://doi.org/10.1109/JBHI.2018.2878846

Bosse, T., Gerritsen, C., de Man, J., Treur, J., 2014. Towards virtual training of emotion regulation. Brain Inform 1, 27-37. https://doi.org/10.1007/s40708-014-0004-9

Botella, C., Bretón-López, J., Quero, S., Baños, R., García-Palacios, A., 2010. Treating Cockroach Phobia With Augmented Reality. Behavior Therapy 41, 401-413. https://doi.org/10.1016/j.beth.2009.07.002

Boyce, P.R., Eklund, N.H., Hamilton, B.J., Bruno, L.D., 2000. Perceptions of safety at night in different lighting conditions. International Journal of Lighting Research and Technology 32, 79-91. https://doi.org/10.1177/0960327100032002 05

Bradley, M.M., Lang, P.J., 1994. Measuring emotion: The self-assessment manikin and the semantic differential. Journal of Behavior Therapy and Experimental Psychiatry 25, 49-59. https://doi.org/10.1016/00057916(94)90063-9

Bratman, G.N., Anderson, C.B., Berman, M.G., Cochran, B., de Vries, S., Flanders, J., Folke, C., Frumkin, H., Gross, J.J., Hartig, T., Kahn, P.H., Kuo, M., Lawler, J.J., Levin, P.S., Lindahl, T., Meyer-Lindenberg, A., Mitchell, R., Ouyang, Z., Roe, J., Scarlett,
L., Smith, J.R., van den Bosch, M., Wheeler, B.W., White, M.P., Zheng, H., Daily, G.C., 2019. Nature and mental health: An ecosystem service perspective. Science Advances 5. https://doi.org/10.1126/sciadv.aax0903

Bratman, G.N., Hamilton, J.P., Daily, G.C., 2012. The impacts of nature experience on human cognitive function and mental health: Nature experience, cognitive function, and mental health. Annals of the New York Academy of Sciences 1249 , 118-136. https://doi.org/10.1111/j.17496632.2011.06400.x

Browning, M.H.E.M., Mimnaugh, K.J., van Riper, C.J., Laurent, H.K., LaValle, S.M., 2020. Can Simulated Nature Support Mental Health? Comparing Short, Single-Doses of 360-Degree Nature Videos in Virtual Reality With the Outdoors. Front. Psychol. 10. https://doi.org/10.3389/fpsyg.2019.02667

Buckley, R., Westaway OAM, D., 2021. Women report that nature tourism provides recovery from psychological trauma. Tourism Recreation Research 1-5.

Dunn, N., Edensor, T., 2020. Rethinking Darkness: Cultures, Histories, Practices. Routledge.

Felnhofer, A., Kothgassner, O.D., Schmidt, M., Heinzle, A.-K., Beutl, L., Hlavacs, H., Kryspin-Exner, I., 2015. Is virtual reality emotionally arousing? Investigating five emotion inducing virtual park scenarios. International Journal of Human-Computer Studies 82, 48-56. https://doi.org/10.1016/j.ijhcs.2015.05.004

Ferrer-García, M., Gutiérrez-Maldonado, J., 2011. The use of virtual reality in the study, assessment, and treatment of body image in eating disorders and nonclinical samples: A review of the literature. Body image 9, 1-11. https://doi.org/10.1016/j.bodyim.2011.10.00 1

Gould van Praag, C.D., Garfinkel, S.N., Sparasci, O., Mees, A., Philippides, A.O., Ware, M., Ottaviani, C., Critchley, H.D., 2017. Mindwandering and alterations to default mode network connectivity when listening to naturalistic versus artificial sounds. Scientific Reports 7, 45273. https://doi.org/10.1038/srep45273

Gross, J.J., Levenson, R.W., 1995. Emotion elicitation using films. Cognition and Emotion 9, 87-108. https://doi.org/10.1080/0269993950840896 6

Herrero, R., García-Palacios, A., Castilla, D., Molinari, G., Botella, C., 2014. Virtual Reality for the Induction of Positive Emotions in the Treatment of Fibromyalgia: 
A Pilot Study over Acceptability, Satisfaction, and the Effect of Virtual Reality on Mood. Cyberpsychology, Behavior, and Social Networking 17, 379384.

https://doi.org/10.1089/cyber.2014.0052

Juan, M.C., Baños, R., Botella, C., Pérez, D., Alcaníiz, M., Monserrat, C., 2006. An Augmented Reality System for the Treatment of Acrophobia: The Sense of Presence Using Immersive Photography. Presence: Teleoperators and Virtual Environments 15, 393-402. https://doi.org/10.1162/pres.15.4.393

Mason, P., 2000. Zoo tourism: The need for more research. Journal of sustainable tourism 8 , 333-339.

McLay, R., Ram, V., Murphy, J., Spira, J., Wood, D.P., Wiederhold, M.D., Wiederhold, B.K., Johnston, S., Reeves, D., 2014. Effect of Virtual Reality PTSD Treatment on Mood and Neurocognitive Outcomes.

Cyberpsychology, Behavior, and Social Networking 17, 439-446. https://doi.org/10.1089/cyber.2013.0383

Mehra, R., Sharma, V.S., Kaulgud, V., Podder, S., 2019. Fostering positive affects in software development environments using extended reality, in: Proceedings of the 4th International Workshop on Emotion Awareness in Software Engineering, SEmotion '19. IEEE Press, Montreal, Quebec, Canada, pp. 42-45. https://doi.org/10.1109/SEmotion.2019.000 16

Parr, H., 2007. Mental health, nature work, and social inclusion. Environment and Planning D: Society and Space 25, 537-561.

Philippot, P., 1993. Inducing and assessing differentiated emotion-feeling states in the laboratory. Cognition and Emotion 7, 171193.

https://doi.org/10.1080/0269993930840918 3

Plante, T.G., Aldridge, A., Bogden, R., Hanelin, C., 2003. Might virtual reality promote the mood benefits of exercise? Computers in Human Behavior 19, 495-509. https://doi.org/10.1016/S07475632(02)00074-2

Riva, G., Mantovani, F., Capideville, C.S., Preziosa, A., Morganti, F., Villani, D., Gaggioli, A., Botella, C., Alcañiz, M., 2007. Affective Interactions Using Virtual Reality: The Link between Presence and Emotions. CyberPsychology \& Behavior 10, 45-56. https://doi.org/10.1089/cpb.2006.9993

Serrano, B., Baños, R.M., Botella, C., 2016. Virtual reality and stimulation of touch and smell for inducing relaxation: A randomized controlled trial. Computers in Human
Behavior 55, 1-8. https://doi.org/10.1016/j.chb.2015.08.007

Thayer, R.E., 1986. Activation-Deactivation Adjective Check List: Current Overview and Structural Analysis. Psychol Rep 58, 607-614. https://doi.org/10.2466/pr0.1986.58.2.607

Yin, J., Yuan, J., Arfaei, N., Catalano, P.J., Allen, J.G., Spengler, J.D., 2020. Effects of biophilic indoor environment on stress and anxiety recovery: A between-subjects experiment in virtual reality. Environment International 136, 105427. https://doi.org/10.1016/j.envint.2019.10542 7

Yu, C.-P., Lee, H.-Y., Luo, X.-Y., 2018. The effect of virtual reality forest and urban environments on physiological and psychological responses. Urban Forestry \& Urban Greening 35, 106-114. https://doi.org/10.1016/j.ufug.2018.08.013 